\title{
Patterns of Opioid and Benzodiazepine Use in Opioid-Naïve Patients with Newly Diagnosed Low Back and Lower Extremity Pain
}

\author{
Tej D. Azad, $M S^{7}$, Yi Zhang, $A B^{7}$, Martin N. Stienen, $M D^{1,2}$, Daniel Vail, $B A^{7}$, \\ Jason P. Bentley, PhD ${ }^{3}$, Allen L. Ho, MD ${ }^{7}$, Paras Fatemi, $M D^{7}$, Daniel Herrick, $M D, P h D^{7}$, \\ Lily H. Kim, BA ${ }^{7}$, Austin Feng, BA ${ }^{7}$, Kunal Varshneya, $B A^{7}$, Michael Jin, $B A^{7}$, \\ Anand Veeravagu, $M D^{7}$, Jayanta Bhattacharya, $M D, P h D^{3}$, Manisha Desai, $P h D^{3}$, \\ Anna Lembke, MD ${ }^{4}$, and John K. Ratliff, $M D^{7}$
}

'Department of Neurosurgery, Stanford University School of Medicine, Stanford, CA, USA; ${ }^{2}$ Department of Neurosurgery \& Clinical Neuroscience Center, University Hospital Zurich, University of Zurich, Zurich, Switzerland; ${ }^{3}$ Quantitative Sciences Unit, Stanford University School of Medicine, Stanford, CA, USA; ${ }^{4}$ Department of Psychiatry and Behavioral Sciences, Stanford University School of Medicine, Stanford, CA, USA.

BACKGROUND: The morbidity and mortality associated with opioid and benzodiazepine co-prescription is a pressing national concern. Little is known about patterns of opioid and benzodiazepine use in patients with acute low back pain or lower extremity pain.

OBJECTIVE: To characterize patterns of opioid and benzodiazepine prescribing among opioid-naïve, newly diagnosed low back pain (LBP) or lower extremity pain (LEP) patients and to investigate the relationship between benzodiazepine prescribing and long-term opioid use.

DESIGN/SETTING: We performed a retrospective analysis of a commercial database containing claims for more than 75 million enrollees in the USA.

PARTICIPANTS: Participants were adult patients newly diagnosed with LBP or LEP between 2008 and 2015 who did not have a red flag diagnosis, had not received an opioid prescription in the 6 months prior to diagnosis, and had 12 months of continuous enrollment after diagnosis.

MAIN OUTCOMES AND MEASURES: Among patients receiving at least one opioid prescription within 12 months of diagnosis, we defined discrete patterns of benzodiazepine prescribing-continued use, new use, stopped use, and never use. We tested the association of these prescription patterns with long-term opioid use, defined as six or more fills within 12 months.

RESULTS: We identified 2,497,653 opioid-naïve patients with newly diagnosed LBP or LEP. Between 2008 and 2015, 31.9\% and $11.5 \%$ of these patients received opioid and benzodiazepine prescriptions, respectively, within 12 months of diagnosis. Rates of opioid prescription decreased from $34.8 \%$ in 2008 to $27.0 \%$ in $2015(P<0.001)$; however, prescribing of benzodiazepines only decreased from $11.6 \%$ in 2008 to $10.8 \%$ in 2015 . Patients with continued or new benzodiazepine use consistently used more opioids than patients who never used or stopped using

Electronic supplementary material The online version of this article (https://doi.org/10.1007/s11606-019-05549-8) contains supplementary material, which is available to authorized users.

Received March 25, 2019

Revised August 7, 2019

Accepted October 24, 2019

Published online November 12, 2019 benzodiazepines during the study period (one-way ANOVA, $P<0.001)$. For patients with continued and new benzodiazepine use, the odds ratio of long-term opioid use compared with those never prescribed a benzodiazepine was 2.99 (95\% CI, 2.89-3.08) and 2.68 (95\% CI, 2.622.75), respectively.

LIMITATIONS: This study used administrative claims analyses, which rely on accuracy and completeness of diagnostic, procedural, and prescription codes.

CONCLUSION: Overall opioid prescribing for low back pain or lower extremity pain decreased substantially during the study period, indicating a shift in management within the medical community. Rates of benzodiazepine prescribing, however, remained at approximately $11 \%$. Concurrent prescriptions of benzodiazepines and opioids after LBP or LEP diagnosis were associated with increased risk of long-term opioid use.

KEY WORDS: low back pain; opioid; benzodiazepine; long-term opioid use.

J Gen Intern Med 35(1):291-7

DOI: $10.1007 / \mathrm{s} 11606-019-05549-8$

(c) Society of General Internal Medicine 2019

\section{INTRODUCTION}

The opioid epidemic represents a major health crisis in the USA, with opioids involved in $66.4 \%$ of drug overdose deaths in 2016. ${ }^{1}$ Opioid and benzodiazepine co-prescribing increases the risk of mortality, ${ }^{2}$ and one-third of fatal opioid overdoses involves a benzodiazepine. ${ }^{3,4}$ Significant concerns about concurrent opioid and benzodiazepine use have been raised, resulting in a 2016 FDA black box warning about the risks of simultaneous opioid and benzodiazepine use. ${ }^{5-7}$ Both opioids and benzodiazepines are prescribed for management of acute low back pain (LBP) or lower extremity pain (LEP), though evidence to support these medications for this indication is limited, with benzodiazepines demonstrated to be ineffective for radicular LBP and insufficient evidence to demonstrate efficacy for non-radicular LBP. ${ }^{8,9}$ 
In a recent study of commercially insured patients, Sun et al. observed that concurrent opioid and benzodiazepine prescribing increased by nearly $80 \%$ from 2001 to 2013. Moreover, the authors observed that patients prescribed opioids who also received benzodiazepine prescriptions were at significantly higher risk of presenting to the emergency department or requiring inpatient admission for opioid overdose. ${ }^{6}$ Ladapo et al. found higher rates of initial opioid prescriptions among patients already prescribed benzodiazepines, compared with the general population. ${ }^{10}$ Complementing these observations, Hernandez et al. observed concurrent opioid and benzodiazepine use was associated with a fivefold increase in the risk of opioid-related overdose during the initial 90 days of concurrent use. ${ }^{5}$ Although LBP and LEP represent common reasons for opioid and benzodiazepine prescriptions in the US population, and such prescriptions are not recommended by guidelines for LBP and LEP management, little is known about patterns of opioid and benzodiazepine use in patients with newly diagnosed LBP or LEP. ${ }^{11-13}$

Given that LBP is one of the most common reasons for seeking medical care and is often managed with both opioids and benzodiazepines, we sought to characterize opioid and benzodiazepine prescribing patterns among patients with newly diagnosed LBP or LEP in the USA.

\section{METHODS}

\section{Data}

We obtained a sample of the MarketScan Commercial Claims and Encounters database (Truven Health Analytics, Ann Arbor, MI) from 1 January 2007 to 31 December 2016, inclusive. This database is a collection of commercial inpatient, outpatient, and pharmaceutical claims of more than 75 million employees, retirees, and dependents representing a substantial portion of the US population covered by employer-sponsored insurance. The MarketScan database contains International Classification of Diseases, 9th Revision, Clinical Modification (ICD-9-CM) and 10th revision, Clinical Modification (ICD10-CM), Current Procedural Terminology (CPT), DiagnosisRelated Group (DRG) codes, and National Drug Codes (NDC). The MarketScan database is commonly used for health care analyses, including investigations of opioid and benzodiazepine use. ${ }^{6,14-17}$ We identified prescriptions from pharmaceutical claims data using variables mapped from the American Hospital Formulary Service Classification Compilation Therapeutic Class. ${ }^{18}$

\section{Sample}

Our sample consisted of 2,497,653 patients (age 18 years or older) who were newly diagnosed with LBP or LEP between 2008 and 2015 and were opioid-naïve for at least 6 months before the diagnosis. This look-back period was selected to be consistent with multiple prior studies of opiate-naïve populations using various administrative claims databases. ${ }^{19-}$ 21 The initial visit was defined as the first encounter at which an enrollee had a claim with an ICD-9-CM or ICD-10-CM code that met the criteria of LBP or LEP (Supplementary Table 1), without any of these inclusion diagnoses in the 12 months prior to this visit. A 12-month continuous enrollment window prior to and after the initial visit was required and we censored all study participants at 12 months following LBP or LEP diagnosis. Patients with a "red flag" LBP diagnosis (e.g., cauda equina syndrome) at any point in the 12 months prior to or following the initial visit (Supplementary Table 1) were excluded. These red flag diagnoses and exclusion criteria were created to minimize the misclassification of chronic LBP or LEP as new LBP or LEP, and to minimize confounders, such as comorbid diagnoses for which patients might receive opioids. A diagram of cohort construction is provided in Supplementary Figure 1.

\section{Outcomes}

Our primary outcome was long-term opioid use, defined as six or more fills within 12 months, consistent with prior studies. ${ }^{22}$, 23 Deyo et al. observed that patients meeting this definition were nearly seven times more likely to be hospitalized due to opioid-related adverse events. ${ }^{22}$

\section{Variables}

The indicator variable of interest for our study was the postdiagnosis pattern of benzodiazepine use in newly diagnosed, opioid-naive LBP and LEP patients. We defined this variable for patients who received at least one opioid prescription $(N=$ 796,568 ) by determining four variable levels representing all combinations of pre-diagnosis and post-diagnosis benzodiazepine use:

1. Continued benzodiazepine use-patients who had received a benzodiazepine prescription in the 6 months preceding LBP or LEP diagnosis and who received at least one new benzodiazepine prescription in the 12 months following diagnosis.

2. New benzodiazepine use - patients who had not received a benzodiazepine prescription in the 6 months preceding LBP or LEP diagnosis and who received at least one new benzodiazepine prescription in the 12 months following diagnosis.

3. Stopped benzodiazepine use-patients who had received a benzodiazepine prescription in the 6 months preceding LBP or LEP diagnosis but who did not receive a new benzodiazepine prescription in the 12 months following diagnosis.

4. Never benzodiazepine use - patients who did not receive a benzodiazepine prescription in the 6 months preceding LBP or LEP diagnosis or in the 12 months following.

In an effort to avoid assuming adherent medication use, we did not specifically compute temporal overlap between opioid 
and benzodiazepine prescriptions. While others adopted an approach of identifying the specific prescription overlap intervals (e.g., defining concurrent use as having at least 1 day of overlap in a given calendar year), ${ }^{6,7}$ we posit that patients are unlikely to adopt patterns of use that strictly adhere to prescription instructions.

Patient-level covariates including age at diagnosis, sex, geographic region, and insurance plan type were taken directly from the claims data at the time of LBP or LEP diagnosis. Comorbidities were ascertained using all inpatient and outpatient records available in the 12 months prior to diagnosis according to the Elixhauser Comorbidity Index. ${ }^{24}$ Low back pain interventions were identified using CPT codes for physical therapy, chiropractic manipulative therapy, epidural steroid injections (ESIs), and spinal surgery. Early opioid prescription was defined as a dispense within 14 days of initial diagnosis, which does not align with standard of care. ${ }^{9}$ Previous studies have demonstrated that early opioid prescription is associated with increased risk for surgery and continued opioid use, despite no significant difference in severity of injury. ${ }^{25}$

\section{Analyses}

We began by calculating the annual percentages of opioid prescription, benzodiazepine prescription, early opioid prescription, and long-term opioid use. The denominator for each of these calculations was the total sample size of LBP or LEP patients per year. The Jonckheere-Terpstra test was used to assess the significance of the decreasing trends in opioid and benzodiazepine prescription between 2008 and 2015. We then sought to describe monthly morphine milligram equivalents (MMEs) among LBP or LEP patients receiving at least one opioid prescription following the day of diagnosis. We tabulated MMEs for each level of our key indicator variable, pattern of benzodiazepine use. One-way ANOVA with Tukey's honestly significant difference (HSD) test was used to assess for significant differences in monthly MMEs between the levels of the benzodiazepine use pattern variable. $P$ values were interpreted as significant only after applying the Bonferroni correction for multiple comparisons.

Finally, we hypothesized that these patterns of benzodiazepine prescribing were associated with long-term opioid use in patients who received at least one opioid prescription in the 12 months following diagnosis. To test this hypothesis, we used multivariable logistic regression to estimate relationship between patterns of benzodiazepine use and long-term opioid use. The key independent variable was a categorical variable for pattern of benzodiazepine use, with "Never benzodiazepine use" as the reference. The model controlled for continuous age, geographic region, sex, insurance plan type, and Elixhauser comorbidities, including psychiatric diagnoses. Comorbidities were included in the model as indicator variables. Results were expressed as odds ratios (ORs) with $95 \%$ confidence intervals (CIs).

\section{Patient Involvement}

As this study includes only analysis of secondary de-identified data, human subject research was not considered and received exemption from the institutional review board approval at our institution.

\section{RESULTS}

\section{Cohort Overview}

Our sample of opioid-naïve patients with newly diagnosed low back or lower extremity pain $(N=2,497,653)$ was $55.0 \%$ female and had a mean age of 47.4 . The most common comorbidities were hypertension $(24.1 \%)$, diabetes mellitus (9.5\%), depression $(7.8 \%)$, chronic pulmonary disease (7.4\%), and hypothyroidism (7.3\%). The prevalence of other psychiatric comorbidities assessed in this population was psychoses $(0.39 \%)$, drug abuse $(0.46 \%)$, and alcohol abuse $(0.61 \%)$. We observed that nearly one in three patients $(31.9 \%)$ received an opioid prescription and more than one in ten $(11.5 \%)$ received a benzodiazepine prescription in the 12 months following initial LBP or LEP diagnosis. Roughly $20 \%$ of patients received physical therapy, $12.2 \%$ underwent chiropractic manipulative therapy, $4.0 \%$ received ESIs, and $1.2 \%$ of patients underwent spine surgery (Table 1 ).

\section{Trends in Opioid and Benzodiazepine Prescribing from 2008 to 2015}

Given the requirement that patients included in our sample had to be continuously enrolled for 1 year prior to LBP or LEP diagnosis and for 1 year following diagnosis, we were able to observe annual trends in pharmacotherapy of opioid-naïve, newly diagnosed LBP or LEP patients from 2008 to 2015. We observed a steady decline in opioid prescribing over the course of our study, from $34.8 \%$ in 2008 to $27.0 \%$ in 2015 $(P<0.001)$. This decline was mirrored by decreasing rates of early opioid prescribing (16.3 to $11.4 \%$ ) and long-term opioid

Table 1 Cohort Overview of Opioid-Naïve Patients with Newly Diagnosed Low Back or Lower Extremity Pain $(N=2,497,653)$

\begin{tabular}{lll}
\hline \hline \multicolumn{1}{c}{$\begin{array}{c}\text { Female } \\
\text { Age }\end{array}$} & N or mean & \% or SD \\
\hline \multicolumn{1}{c}{$\begin{array}{l}\text { Ag, } \\
\text { Comorbidities }\end{array}$} & 47.4 & $55.0 \%$ \\
Hypertension & 601,317 & 15.8 \\
Diabetes mellitus & 238,165 & $24.1 \%$ \\
Depression & 194,401 & $9.5 \%$ \\
Chronic pulmonary disease & 185,782 & $7.8 \%$ \\
Hypothyroidism & 181,858 & $7.4 \%$ \\
12-month pharmacotherapy & 796,568 & $7.3 \%$ \\
Opioid & 286,457 & $31.9 \%$ \\
Benzodiazepine & 375,862 & $11.5 \%$ \\
Early opioid prescription & & $15.1 \%$ \\
12-month interventions & 509,367 & $20.4 \%$ \\
Physical therapy & 304,505 & $12.2 \%$ \\
Chiropractic manipulative therapy & 99,222 & $4.0 \%$ \\
Epidural steroid injection & 30,604 & $1.2 \%$ \\
Surgery & & \\
\hline
\end{tabular}


use (2.7 to $1.2 \%$ ). Rates of benzodiazepine prescribing did not appear to change markedly: $11.6 \%$ in 2008 to $10.7 \%$ in 2015

$$
(P=0.031)(\text { Fig. 1).] } \rightarrow
$$

\section{Benzodiazepine Prescription Correlates with Higher Mean Monthly Opioid Use}

We characterized patterns of benzodiazepine prescribing among patients who received at least one opioid prescription in the year following diagnosis $(N=796,568)$. Overall, $18.3 \%$ of patients $(N=145,411)$ who received an opioid prescription in the year following diagnosis $(N=796,568)$ also filled a benzodiazepine prescription during this period. Patients were categorized into four groups on the basis of pre- and postdiagnosis benzodiazepine prescription: continued benzodiazepine use ( $>1$ prescription filled in the 6 months before diagnosis and in the 12 months after, $N=50,763$ ), new benzodiazepine use (no prescription filled in the 6 months before diagnosis but $>1$ filled in the 12 months after, $N=94,648$ ), stopped benzodiazepine use ( $>1$ prescription filled in the 6 months before diagnosis but no prescriptions filled in the 12 months after, $N=14,771$ ), and never benzodiazepine use (no prescription filled pre- or post-diagnosis, $N=636,386$ ). In the year following diagnosis, patients in the continued benzodiazepine use group used on average 1577 MMEs (standard error of the mean (SEM), 25.8), patients in the new benzodiazepine use group used on average 1589 MMEs (SEM, 20.2), and patients in the stopped benzodiazepine use group used on average 1094 MMEs (SEM, 41.3), while patients in the never benzodiazepine use group used on average 830 MMEs (SEM, 5.1) (one-way ANOVA, $P<0.001$ ).

We then calculated the mean monthly MMEs of each group, for each month following diagnosis (Fig. 2). We observed that LBP or LEP patients who received at least one opioid prescription and had continued or new benzodiazepine use were consistently prescribed higher amounts of opioids compared with patients who stopped benzodiazepine use or who never used benzodiazepines. One-way ANOVA, with correction for multiple hypotheses, was significant for each month following diagnosis $(P<0.001)$.] $\rightarrow$

\section{Continuous or New Benzodiazepine Prescribing Is Associated with Longer Term Opioid Prescribing}

Using a multivariable logistic regression model adjusted for age, sex, insurance type, geographic region, and Elixhauser comorbidities (including psychiatric diagnoses of depression, psychoses, drug abuse, and alcohol abuse), we found that, compared with patients who never received a benzodiazepine prescription, those in the stopped benzodiazepine use group were at somewhat higher risk of long-term opioid use (OR, $1.40 ; 95 \%$ CI, 1.30-1.50; $P<0.001)$, while patients in the new benzodiazepine use (OR, 2.69; 95\% CI, 2.62-2.75; $P<0.001$ ) and continued benzodiazepine use (OR, 2.99; 95\% CI, 2.89$3.08 ; P<0.001)$ groups were at markedly higher risk of longterm opioid use (Fig. 3).] $\rightarrow$

\section{DISCUSSION}

Opioid prescribing declined from 34.8 to $27.0 \%$ during the time period assessed (2008 to 2015), indicating a shift in the management of acute low back pain within our cohort. Benzodiazepine prescribing within the same cohort remained at approximately $11 \%$ within the study period. Benzodiazepine prescribing was associated with long-term opioid use compared with those who had never received a benzodiazepine prescription.

Previous studies have demonstrated that, despite the risks of concomitant opioid and benzodiazepine prescriptions, opioid

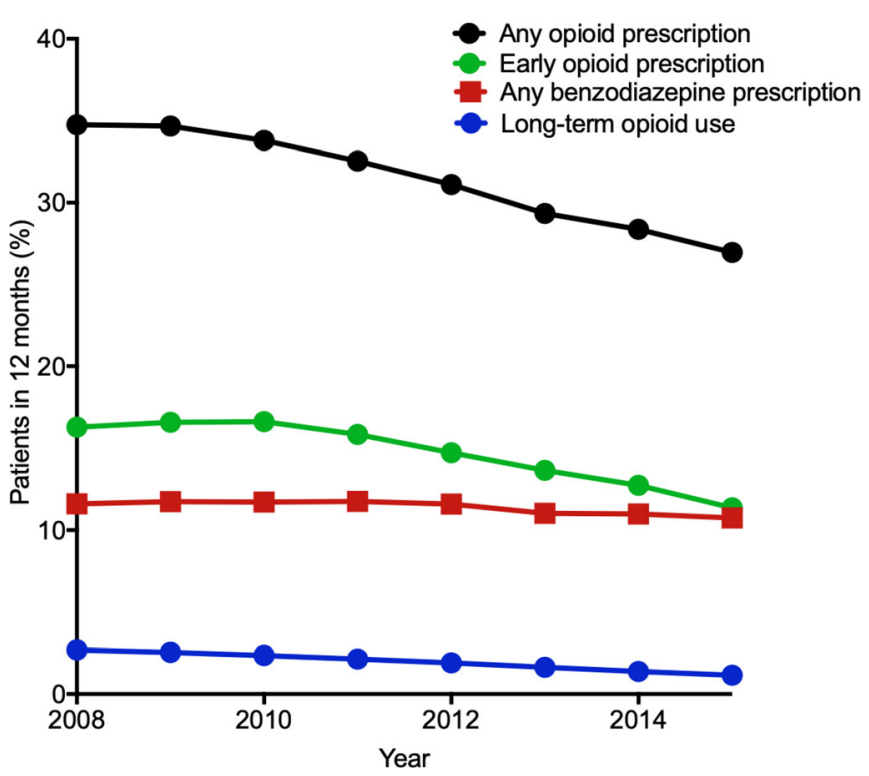

Fig. 1 Trends in opioid and benzodiazepine prescribing over time among commercially insured patients in the USA. 


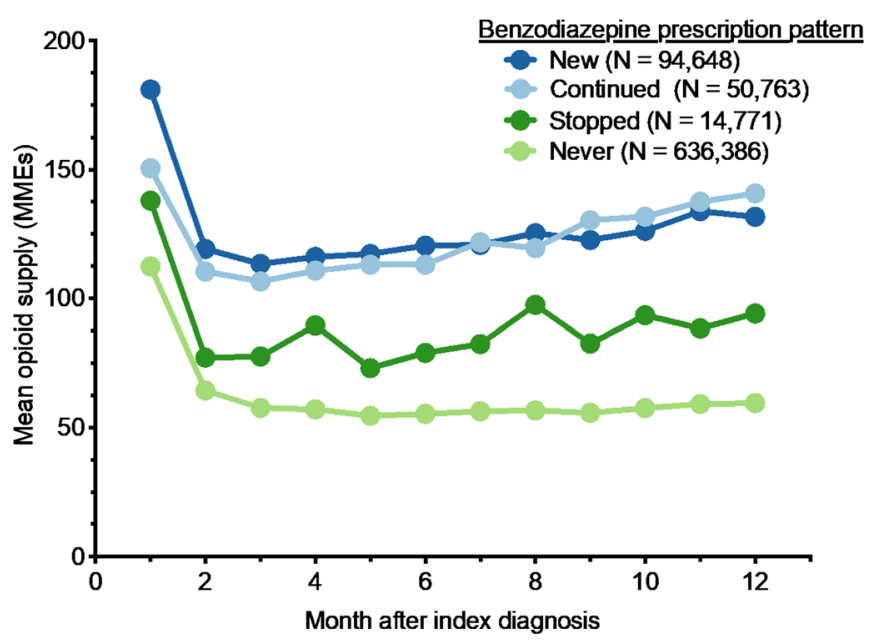

Fig. 2 Opioid use varies with benzodiazepine prescribing pattern among following LBP diagnosis. Graphs illustrating that opioid use (y-axis: mean milligram morphine equivalents, MMEs) varies with benzodiazepine prescribing over time ( $x$-axis: months after LBP diagnosis). The data is limited to patients who received at least one opioid prescription during the study period $(\mathrm{N}=796,568)$.

and benzodiazepine co-prescribing is not uncommon. A 2015 cross-sectional analysis showed that benzodiazepines were prescribed with opioids at $8.1 \%$ and $15 \%$ of acute and chronic musculoskeletal pain visits, respectively. ${ }^{26}$ A 2016 prescription database study reported that the annual proportion of patients receiving opioids who also received a benzodiazepine increased from 6.8 to $9.6 \%$ between 2002 and 2014. ${ }^{27}$ Our results confirm that the pharmacological management of LBP or LEP tracks these general prescribing patterns. In this cohort, $18.2 \%$ of LBP or LEP patients who received an opioid prescription within 12 months of initial diagnosis also filled a benzodiazepine prescription at some point during the 12month period. Reasons for co-prescribing may include provider beliefs that benzodiazepines provide quick and effective relief of muscle spasms, anxiety, insomnia, and other painassociated symptoms, despite a lack of strong evidence supporting these beliefs. ${ }^{27}$

This rate of opioid and benzodiazepine co-prescribing is concerning, given the dangers associated with using opioids and benzodiazepines concurrently. Furthermore, we demonstrate that while the frequency of opioid prescriptions for LBP or LEP has steadily declined in recent years, rates of benzodiazepine prescriptions have declined by a much smaller magnitude throughout the study period. While opioid prescribing patterns have benefitted from significant attention and policy interventions in recent years, benzodiazepine prescriptions have not received the same scrutiny. Medicare's recent decision to implement a point-of-sale soft edit that prevents filling of concurrent opioid and benzodiazepine prescriptions written by different providers, unless overridden by the pharmacist, is an important step in this direction. ${ }^{28}$ Additional emphasis on minimizing opioid and benzodiazepine co-prescriptions, particularly for indications such as LBP for which data demonstrating efficacy remains limited, may be an opportunity to further improve prescribing patterns. ${ }^{2,8,9}$

Concurrent use of benzodiazepines is known to increase risk of opioid-related adverse events, for instance, by increasing or prolonging the respiratory depression produced by opioid medications. ${ }^{29}$ Our study demonstrates that patients with continued or new prescription of benzodiazepines

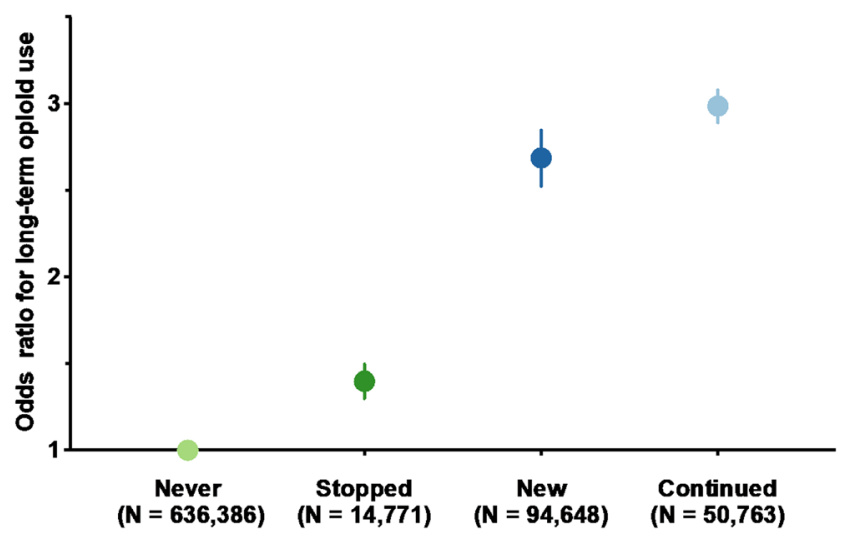

Pattem of benzodiazepine use in newly-diagnosed opiate-naïve LBP patients

Fig. 3 Association of benzodiazepine prescribing with long-term opioid use. Graph illustrating the relationship between benzodiazepine prescription type $(x$-axis) and the likelihood for meeting the definition of long-term opioid use (y-axis). Results are expressed as odds ratios (point) and 95\% confidence intervals (bars), with adjustment for age, sex, insurance type, region, and Elixhauser comorbidities. 
following LBP or LEP diagnosis are at higher risk of longterm opioid use.

This study had several limitations. We used administrative claims analyses, which rely on accuracy of diagnostic, procedural, and prescription codes. While our model controlled for some comorbid psychiatric diagnoses, including alcohol abuse, drug abuse, psychoses, and depression, it is possible that some patients receiving benzodiazepine prescriptions may have been receiving them in the management of mental health disorders that are associated with increased risk of opioid dependence. Finally, although opioid prescriptions are likely to be prescribed repeatedly only if they are taken by the patient, claims data details medications that were prescribed and dispensed to the patient, but does not account for medications that were filled but not taken, medications obtained from sources not covered by the patient's insurance, and does not allow us to determine precisely that the medications were prescribed for LBP or LEP.

Further study might elucidate the relationship between opioid-benzodiazepine co-prescription and opioid use by examining the association between specific benzodiazepine prescription patterns, such as use of shorter-acting benzodiazepines or dose augmentation, and increased risk of long-term opioid use. Additional investigation into the relationship between other medication classes, like gabapentinoids or skeletal muscle relaxants, and risk of long-term opioid use after LBP or LEP diagnosis may be warranted.

\section{CONCLUSION}

Our analysis shows that concomitant prescriptions of benzodiazepines and opioids are associated with higher doses of opioids prescribed and increased frequency of longterm opioid use. The rate of benzodiazepine and opioid co-prescribing has remained high over the past several years, even as overall rates of opioid prescribing have decreased. Our results identify opioid and benzodiazepine co-prescribing as a potential target for policy interventions to improve the safety of pharmacological management of LBP and LEP. Further work could investigate patient and provider factors that lead to co-prescribing of opioids and whether these drugs used in combination impact tolerance, dependence, reinforcement, or aspects of pharmacodynamics that might be leading to increased use.

Corresponding Author: John K. Ratliff, MD; Department of Neurosurgery Stanford University School of Medicine, Stanford, CA, USA (e-mail: jratliff@stanford.edu).

\section{Compliance with Ethical Standards:}

Conflict of Interest: The authors declare that they do not have a conflict of interest.

\section{REFERENCES}

1. Seth P, Scholl L, Rudd RA, Bacon S. Overdose deaths involving opioids, cocaine, and psychostimulants - United States, 2015-2016. MMWR Morb Mortal Wkly Rep. 2018;67(12):349-358.

2. Lembke A, Papac J, Humphreys K. Our other prescription drug problem. The New England journal of medicine. 2018;378(8):693-695.

3. Saunders KW, Von Korff M, Campbell CI, et al. Concurrent use of alcohol and sedatives among persons prescribed chronic opioid therapy: prevalence and risk factors. The journal of pain : official journal of the American Pain Society. 2012;13(3):266-275.

4. Morasco BJ, Duckart JP, Carr TP, Deyo RA, Dobscha SK. Clinical characteristics of veterans prescribed high doses of opioid medications for chronic non-cancer pain. Pain. 2010;151(3):625-632.

5. Hernandez I, He M, Brooks MM, Zhang Y. Exposure-response association between concurrent opioid and benzodiazepine use and risk of opioid-related overdose in Medicare part D beneficiaries. JAMA Netw Open. 2018;1(2):e180919.

6. Sun EC, Dixit A, Humphreys $\mathbf{K}$, Darnall BD, Baker LC, Mackey S. Association between concurrent use of prescription opioids and benzodiazepines and overdose: retrospective analysis. BMJ. 2017;356:j760.

7. Park TW, Saitz R, Ganoczy D, Ilgen MA, Bohnert AS. Benzodiazepine prescribing patterns and deaths from drug overdose among US veterans receiving opioid analgesics: case-cohort study. BMJ. 2015;350:h2698.

8. Chou R, Deyo R, Friedly J, et al. Systemic pharmacologic therapies for low back pain: a systematic review for an American College of Physicians clinical practice guideline. Annals of internal medicine. 2017;166(7):480492.

9. Gaseem A, Wilt TJ, McLean RM, Forciea MA, Clinical Guidelines Committee of the American College of P. Noninvasive treatments for acute, subacute, and chronic low back pain: a clinical practice guideline from the American College of Physicians. Annals of internal medicine. 2017;166(7):514-530.

10. Ladapo JA, Larochelle MR, Chen A, et al. Physician prescribing of opioids to patients at increased risk of overdose from benzodiazepine use in the United States. JAMA Psychiatry. 2018;75(6):623-630.

11. Azad TD, Vail D, Bentley $\mathbf{J}$, et al. Initial provider specialty is associated with long-term opiate use in patients with newly diagnosed low back and lower extremity pain. Spine. 2019;44(3):211-218.

12. Thackeray A, Hess R, Dorius J, Brodke D, Fritz J. Relationship of opioid prescriptions to physical therapy referral and participation for Medicaid patients with new-onset low back pain. J Am Board Fam Med. 2017;30(6):784-794.

13. Gold LS, Hansen RN, Avins AL, et al. Associations of early opioid use with patient-reported outcomes and health care utilization among older adults with low back pain. The Clinical journal of pain. 2018;34(4):297305.

14. Azad TD, Vail D, O'Connell C, Han SS, Veeravagu A, Ratliff JK. Geographic variation in the surgical management of lumbar spondylolisthesis: characterizing practice patterns and outcomes. The spine journal : official journal of the North American Spine Society. 2018;18(12):2232-2238.

15. Paulozzi LJ, Zhou C, Jones CM, Xu L, Florence CS. Changes in the medical management of patients on opioid analgesics following a diagnosis of substance abuse. Pharmacoepidemiol Drug Saf. 2016;25(5):545-552.

16. Dunn A, Liebman E, Pack S, Shapiro AH. Medical care price indexes for patients with employer-provided insurance: nationally representative estimates from MarketScan Data. Health Serv Res. 2013;48(3):11731190.

17. Chernew ME, Sabik LM, Chandra A, Gibson TB, Newhouse JP. Geographic correlation between large-firm commercial spending and Medicare spending. Am J Manag Care. 2010;16(2):131-138.

18. American Hospital Formulary S. AHFS Pharmacologic-Therapeutic Classification. In:2018.

19. Jeffery MM, Hooten WM, Hess EP, et al. Opioid prescribing for opioidnaive patients in emergency departments and other settings: characteristics of prescriptions and association with long-term use. Ann Emerg Med. 2018;71(3):326-336.e319.

20. Barnett ML, Olenski AR, Jena AB. Opioid-prescribing patterns of emergency physicians and risk of long-term use. $N$ Engl $J$ Med. 2017;376(7):663-673.

21. Barnett ML, Zhao $\mathbf{X}$, Fine $\mathbf{M J}$, et al. Emergency physician opioid prescribing and risk of long-term use in the Veterans Health Administration: an observational analysis. J Gen Intern Med. 2019;34(8):1522-1529. 
22. Deyo RA, Hallvik SE, Hildebran C, et al. Association between initial opioid prescribing patterns and subsequent long-term use among opioidnaive patients: a statewide retrospective cohort study. $J$ Gen Intern Med. 2017;32(1):21-27.

23. Morden NE, Munson JC, Colla CH, et al. Prescription opioid use among disabled Medicare beneficiaries: intensity, trends, and regional variation. Med Care. 2014;52(9):852-859.

24. Guan $\mathbf{H}$, Sundararajan V, Halfon $\mathbf{P}$, et al. Coding algorithms for defining comorbidities in ICD-9-CM and ICD-10 administrative data. Med Care. 2005;43(11):1130-1139.

25. Webster BS, Verma SK, Gatchel RJ. Relationship between early opioid prescribing for acute occupational low back pain and disability duration, medical costs, subsequent surgery and late opioid use. Spine. 2007;32(19):2127-2132
26. Larochelle MR, Zhang F, Ross-Degnan D, Wharam JF. Trends in opioid prescribing and co-prescribing of sedative hypnotics for acute and chronic musculoskeletal pain: 2001-2010. Pharmacoepidemiol Drug Saf. 2015;24(8):885-892.

27. Hwang CS, Kang EM, Kornegay CJ, Staffa JA, Jones CM, McAninch JK. Trends in the concomitant prescribing of opioids and benzodiazepines, 2002-2014. Am J Prev Med. 2016;51(2):151-160.

28. Centers for M, Medicaid S. Improving drug utilization review controls in part D. In. Vol 20192019.

29. Gudin JA, Mogali S, Jones JD, Comer SD. Risks, management, and monitoring of combination opioid, benzodiazepines, and/or alcohol use. Postgrad Med. 2013;125(4):115-130.

Publisher's Note Springer Nature remains neutral with regard to jurisdictional claims in published maps and institutional affiliations. 